Research Paper

\title{
Field trial of all-optical 40 Gbps transmission system for next-generation networks
}

\author{
Mikio Yagi ${ }^{1}$, Shinya Tanaka ${ }^{2}$, Shuichi Satomi ${ }^{3}$, Shiro Ryu ${ }^{4}$, \\ Ahmad Suffian Mohamad ${ }^{5}$, Susumu Yoneda ${ }^{6}$, Takayuki Fujino ${ }^{7}$, Shoichiro Asano ${ }^{8}$ \\ 1,2,3,4,6 Information and Communication Laboratories, R\&D Division, Japan Telecom Co., Ltd \\ ${ }^{5}$ University of Tokyo \\ ${ }^{6,7,8}$ National Institute of Informatics
}

\begin{abstract}
The National Institute of Informatics (NII) has been conducting joint research with JapanTelecom to develop a $40 \mathrm{Gbps}$ optical transport system for the next-generation SuperSINET. We report on the recent results of field trials focused on automatic chromatic dispersion compensation and path protection/restoration applied to the future SuperSINET as key technologies required to maintain the quality of transmission, to reduce operational costs, and to facilitate the enhancement of network capabilities. From the results of the field trial, we have confirmed that an automatic optical path control system is essential in an all-optical wavelength path network.
\end{abstract}

\section{KEYWORDS}

Optical communication, chromatic dispersion compensation, protection/restoration, GMPLS, multi-layer integration.

\section{Introduction}

Recently there have been many studies regarding all-optical network technology. In future all-optical networks, it is assumed that wavelength paths will be dynamically reconfigured in cases of network protection/restoration and path switching driven by service applications. Accordingly, we must consider a number of new issues that have not been addressed in terms of traditional networks.

SuperSINET, which began in January 2002 as an optical transport infrastructure for high-end scientific research, has been operating at $10 \mathrm{Gbps}$ transmission speed by deploying wavelength division multiplexing (WDM) and optical cross-connect (OXC, or sometimes referred as photonic cross-connect PXC) to maintain quality and reliability of transmission. However, operation of the transport system is based on stat-

Received December 1, 2004; Accepted December 2, 2004

(1)mikio.yagi, 2)shinya.tanaka, 3)shuichi.satomi, 4)shiro.ryu,

6)susumu.yoneda\}@japan-telecom.co.jp, \{5)suffy, 7)fujino, 8)asano\}@nii.ac.jp ic control; the development of more economical and enhanced control systems based on recent technologies thus poses a challenge in the realization of a nextgeneration SuperSINET.

When the wavelength path in an all-optical network is dynamically reconfigured, the accumulated physical layer parameters such as chromatic dispersion (CD) along the path are changed. Even if a slope-compensating dispersion compensation fiber (SC-DCF) is applied for each fiber span, residual chromatic dispersion remains, due to limitations in production accuracy in terms of the length of the SC-DCF. Moreover, CD characteristics vary according to environmental conditions such as ambient temperature [3]. Hence, it is considered that the residual $\mathrm{CD}$ value along the entire path changes from one path to another and that this value changes with time. Therefore, it is possible that signal quality will be degraded without proper compensation in the residual $\mathrm{CD}$ for data rates over 40 Gbps.

Several studies examining $\mathrm{CD}$ along the entire 
wavelength path have already been published. Reference [4] uses GMPLS signaling to calculate chromatic dispersion over the entire path. This method can only treat the $\mathrm{CD}$ characteristics of the installed fiber, as this method is essentially static. However, ambient temperature changes the characteristics of the installed fiber, as described above. For these reasons, it can be said that after initial CD compensator setup by GMPLS signaling as shown in [4], dynamic CD compensation based on time-to-time measurement of signal quality should be applied.

On the other hand, the control of fiber/wavelength path switching is another essential element in executing dynamic path reconfiguration. Many national research networks (NRNs) have been investigating path control technologies, as the provisioning of the wavelength path must be completed within a few seconds to respond to a dynamic request from a data GRID application, protection and restoration. Even though the generalized multi-protocol label switching (GMPLS) protocol [1],[2] is a promising candidate for wavelength path control, issues concerning integration of path resource management, topology control, re-allocation of existing IP transmission flows, and chromatic dispersion control remain unsolved.

In this paper, we report the results of the field trial of dynamic CD compensation assuming initial CD compensator setup by GMPLS signaling. In the trial, however, initial CD compensator setup by GMPLS signaling was not implemented due to the unavailability of an appropriate system. Only dynamic CD compensation system behavior has thus been evaluated. A 40 Gbps field trial was carried out using field fibers $240 \mathrm{~km}$ in length. In the trial, a network protection function was provided by a photonic cross-connect (PXC) using GMPLS signaling. We applied an automatic CD compensator to compensate for the residual $\mathrm{CD}$ dynamically after the network protection operation.

\section{SuperSNET and Optical Technologies}

There is a growing need for high-speed, high-capacity networks within the academic and research communities, as grid applications become increasingly important. To meet this demand, the e-Japan Priority Policy Program announced by the IT Strategic Headquarters in March 2001 cited the SuperSINET network, one of the most advanced optical Internet for research networks, based on 10 Gbps IP/optical communication technology. SuperSINET is a high-speed network intended to develop and promote Japanese academic research by strengthening collaboration among leading academic research institutes within Japan and around the world. For the time being, the network will be used as a basis for research information connecting universities and research institutes in the five fields of high energy and nuclear fusion; space and astronomical science; genome information analysis (bio-informatics); computing GRID applications; and nanotechnology. The Internet backbone connects research institutes at speeds of $10 \mathrm{Gbps}$, and the leading research facilities in the research institutes are directly connected at 1 Gbps. The IT-Based Laboratory (ITBL) Project is also based on Super SINET.

Currently, IP routers, DWDM/CWDM for lambda transmission, and PXC for fiber/lambda protection switching are installed to connect 30 universities and research institutes. Deployment of high-speed transport systems, such as 40 Gbps systems, as well as dynamic lambda operation in conjunction with GRID demand-based resource management, are important steps in the reduction of operational costs and in increasing flexibility and reliability of the network. These elements are scheduled for incorporation in the next version of SuperSINET, beginning in 2007.

The field trial reported in this paper is aimed to conduct feasibility testing for the coming SuperSINET, including testing of 40 Gbps high-quality transmission using single-mode fiber. Quality adjustment capability will also be tested in case of fiber/lambda switching.

\section{Field Demonstration}

\subsection{Experimental Setup}

Figure 1 shows the cable route used in the field trial [5]. Installed cables in Tokyo area were used to configure a transmission system of up to $240 \mathrm{~km}$. All fibers used in the field trial were single-mode fibers (SMF).

Figure 2 shows the field trial setup. Transmitters were comprised of 24 lasers of wavelengths ranging from $1,538 \mathrm{~nm}$ to $1,559 \mathrm{~nm}$ with a channel spacing of $100 \mathrm{GHz}$ on the ITU grid. The modulation data format was NRZ, at a data rate of 40 Gbps. The transmission line consisted of three spans, as shown in Fig. 1. The second span incorporates another fiber span (Line 290 $\mathrm{km}$ long. Switching can be performed between the original fiber span, $80 \mathrm{~km}$ long (Line 1) and another fiber span (Line 2) using the network protection function of the GMPLS-controlled PXCs (PXC 1 and PXC 2) before and after the second span. Between each span, an optical amplifier repeater is installed; in each repeater the $\mathrm{CD}$ and $\mathrm{CD}$ slope is almost fully compensated with a SC-DCF. The SC-DCF was designed to compensate for a $\mathrm{CD}$ and $\mathrm{CD}$ slope of approximately $80 \mathrm{~km} \mathrm{SMF}$, so if Line 2 is selected for the second span, uncompensated residual CD appears at the receiver end. Additionally, for Line 1 a small amount of residual $\mathrm{CD}$ is observed. 


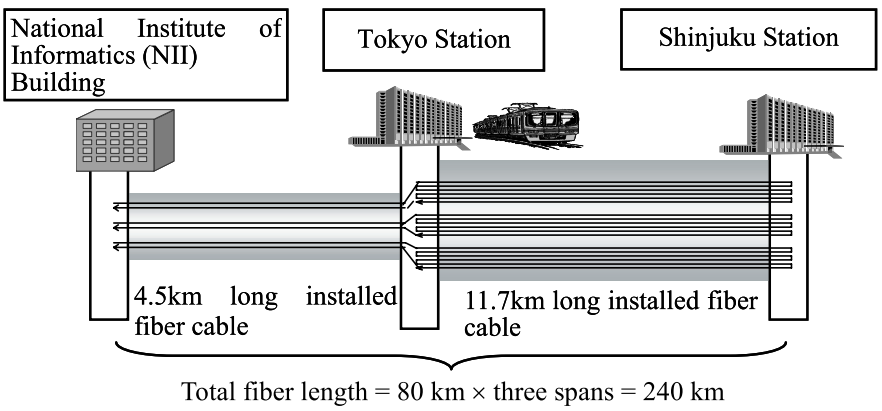

Fig. 1. Field Trial in Tokyo.

40Gbps $\times 24-$ channel transmitters
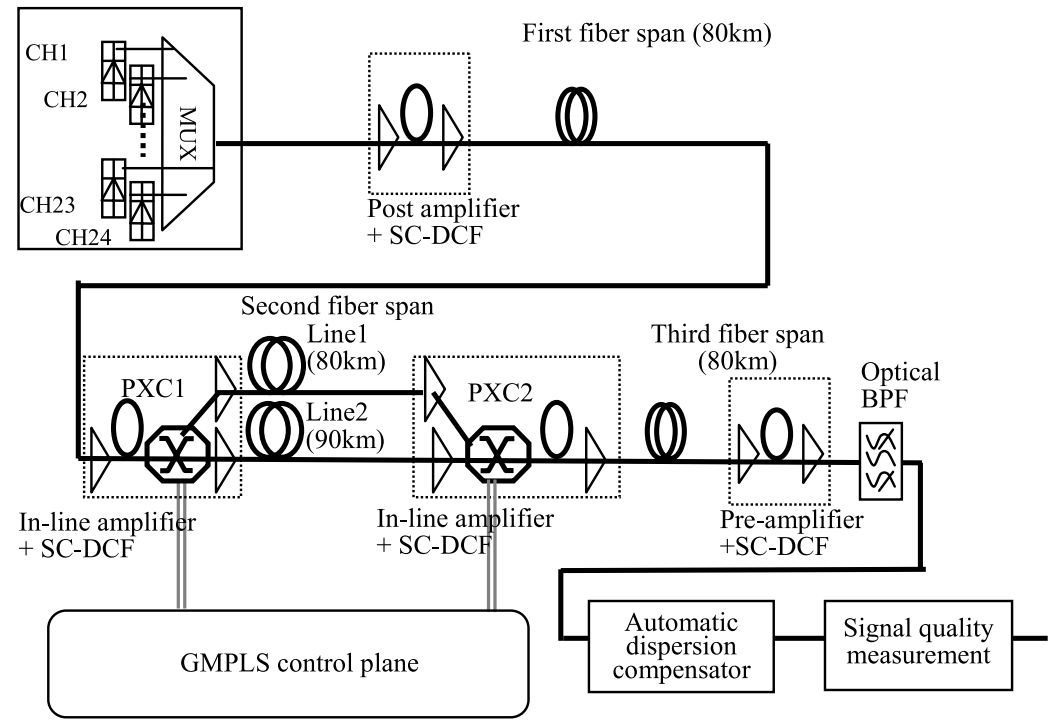

Fig. 2. Field Trial Setup.

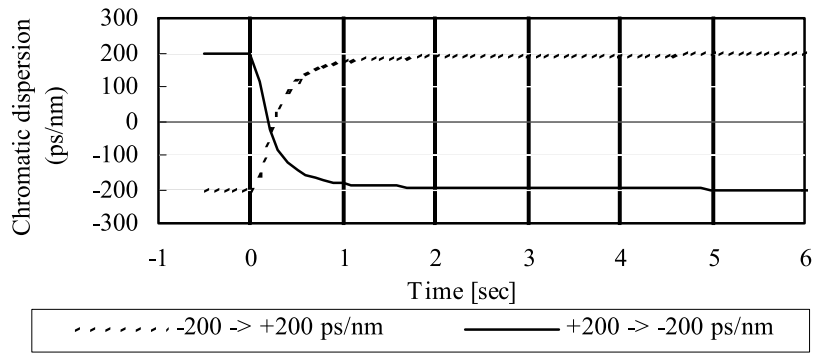

Fig. 3. Response Time of the Chromatic Dispersion ompensator

We applied a fiber Bragg grating (FBG)-based tunable CD compensator device [6] at the receiver. The compensator can compensate for a CD of $-200 \mathrm{ps} / \mathrm{nm}$ $\sim+200 \mathrm{ps} / \mathrm{nm}$ at $1,550 \mathrm{~nm}$, and the CD adjustment time required to reach the target $\mathrm{CD}$ was less than five seconds. Fig. 3 shows the response time of the CD compensator. In this measurement, the value of the chromatic dispersion was changed from $-200 \mathrm{ps} / \mathrm{nm}$ to $+200 \mathrm{ps} / \mathrm{nm}$ and from $+200 \mathrm{ps} / \mathrm{nm}$ to $-200 \mathrm{ps} / \mathrm{nm}$. We can see from these results that this device can set the 


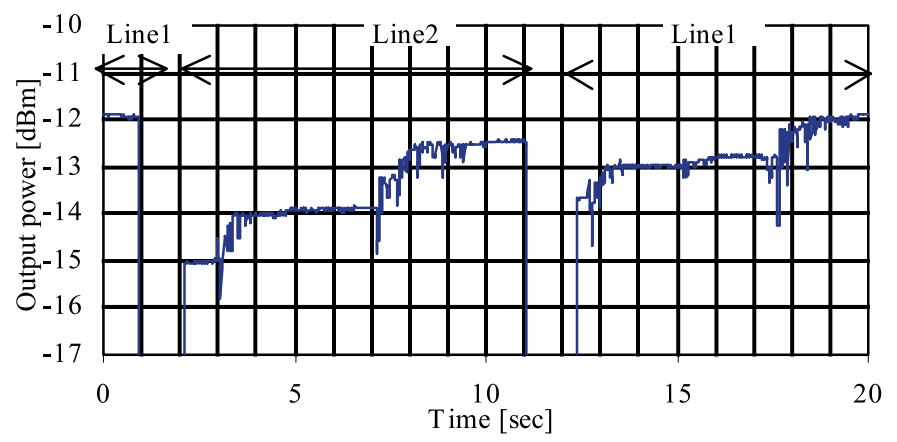

Fig. 4. Protection Performance using PXC.

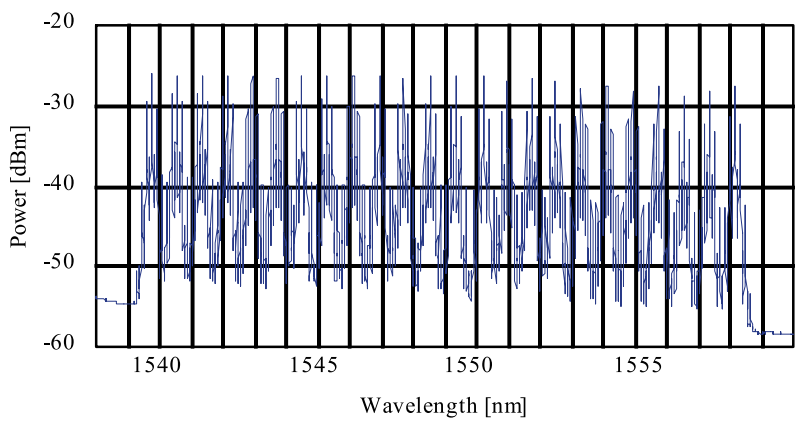

Fig5.a. Optical Spectrum after $240 \mathrm{~km}$ Transmission.

CD target value in about 4.7 seconds. The automatic CD compensator was controlled using the hill-climbing method by monitoring the Q-factor and bit error rate (BER) as transmission quality evaluation parameters [7].

\subsection{Control Plane Operation for Network Protection}

The protection function is based on the GMPLS protocol. The PXCs use the resource reservation protocol with traffic engineering extensions (RSVP-TE) messages [1]. We set up one TE-Link between PXCs with two fibers, Line 1 and Line 2, as data links. In this setup the PXC monitors the optical input and output power. If the power level falls below the threshold, the protection function is initiated. The PXC then indicates the status of the path using an RSVP-NOTIFY message. When the message reaches a path initiator (PXC $1)$, the initiator tears down the path using an RSVPTEAR message; it then sets a new path using an RSVP-PATH/RESV message. The RSVP-TE message flow above is based on the specification of PXCs; we expect that setting up a new path before deleting the existing path may enable faster control.

We observed the protection performance. We injected light into the PXC 1 input port and monitored the optical power at the PXC 2 output port. The results of

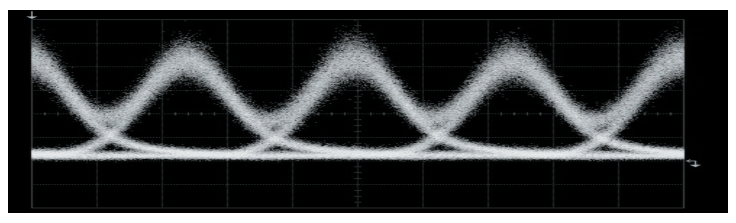

Fig. 5.b. Optical Eye Diagram.

PXC protection performance are shown in Fig. 4. The initial path was Line 1 . When Line 1 was disconnected, the path was switched to Line 2. The network down time due to the protection operation was approximately one second. This period includes the time required for optical power detection, the time required for RSVP-TE signaling, and the time required for optical-switch stabilization. The RSVP-TE signaling time is estimated to be less than approximately $100 \mathrm{~ms}$ and the optical-switch stabilization time is approx. $10 \mathrm{~ms}$. Hence, it is concluded that the relatively long protection time is due to the fact that optical-power detection time, including the guard time for the LOS (Loss of Light) signal, is dominant in the protection operation.

\section{Results and Discussion}

First, we performed transmission experiments without the network protection function. Figure 5(a) and Fig. 5(b) show the optical spectrum and the optical eye diagram after transmission over $240 \mathrm{~km}$ installed fibers, respectively. Optical signal-to-noise ratio (OSNR) was more than $25 \mathrm{~dB}$, and the eye diagram opened up clearly

Next, we performed transmission experiments using the protection function. Residual CD values for the Line 1 and Line 2 cases with the second span were 


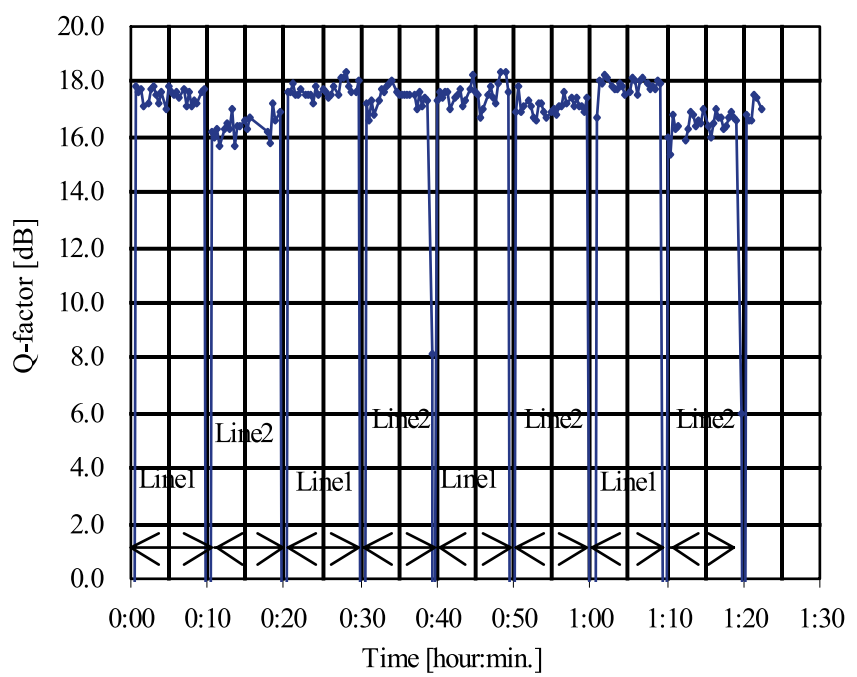

Fig. 6. Outline of $Q$-factor in Network Protection.

-32 and $+147 \mathrm{ps} / \mathrm{nm}$ at $1,550 \mathrm{~nm}$, respectively. The automatic CD compensation experiment in the case of a network protection operation using GMPLS signaling was performed by switching between Line 1 and Line 2 at the second span every 10 minutes.

Figure 6 shows the variation in the Q-factor. As can be seen from this figure, the Q-factor degraded just after the protection occurred and the line was switched, since the residual CD for the new wavelength path reduced the transmission performance of the $40 \mathrm{Gbps}$ signal. However, the Q-factor recovered to a satisfactory value after the automatic $\mathrm{CD}$ compensation system compensated for the residual $\mathrm{CD}$ to obtain optimum performance. The average recovery time for the Q-factor was measured to be approx. 40 seconds. The recovery time includes the time required to measure signal quality and the time required to control the CD compensator device. The ratio of measurement time to control time was approx. 6:4. It is assumed that in a future optical network, dynamic reconfiguration of the network will occur frequently. It is thus expected that rapid optical performance monitoring and a faster CD compensation device will be essential.

Recently numerous research efforts have focused on the electrical CD compensator [8] as well as on the optical CD compensator. Many optical CD compensators, such as FBGs, are operated by temperature control. Temperature control results in increased response time. A faster response time can generally be obtained using an electrical CD compensator. As a result, it can be expected that using an electrical compensator may enable the reduction of response time to approximately several tens of milliseconds. In terms of signal qual- ity measurement, some measurement methods are available that are faster than those employed in this experiment. Through a combination of these CD compensation devices and a signal quality measurement method, we can expect that recovery time will be reduced to within one second.

From the field trial, we have confirmed that $40 \mathrm{Gbps}$ wavelength path service with GMPLS photonic network control is feasible with the application of an automatic CD compensation system.

\section{Conclusion}

We carried out a field trial for $40 \mathrm{Gbps}$ wavelength path protection using PXC and GMPLS control over $240 \mathrm{~km}$ installed fibers in view of the realization of an all-optical network. We applied an automatic CD compensation system to the network. From the results of the field trial, we have concluded that an automatic $\mathrm{CD}$ compensation system is essential in providing demand-based 40Gbps wavelength path provisioning in a next-generation SuperSINET.

We are also conducting further trials to shorten the response time for protection/restoration and to increase the quality of all-optical transport, in order to stimulate scientific applications incorporating computational and data GRID use with extremely high throughput based on nearly error-free transport at 40 Gbps. Results of successive trials will be reported in the near future.

\section{References}

[1] L. Berger, "Generalized Multi-Protocol Label 
Switching (GMPLS) Signaling Resource Reservation Protocol-Traffic Engineering (RSVP-TE) Extensions," RFC3473, IETF.

[2] L. Berger, "Generalized Multi-Protocol Label Switching (GMPLS) Signaling Functional Description," RFC 3471, IETF.

[3] T. Kato, Y. Koyano, and M. Nishimura, "Temperature dependence of chromatic dispersion in various type of optical fibers," Proc. OFC2000, Paper TuG7, March 2000.

[4] T. Hashimoto, M. Yagi, H. Koyano, S. Tanaka, A. Inomata, S. Satomi, H. Ishimatsu, and S. Ryu, "Rapid automatic chromatic dispersion compensation using GMPLS signaling enhancement for dynamically reconfigurable all-optical network,"Proc. ECOC2003, Paper Mo4.7.6, Sept. 2003.

[5] M. Yagi, S. Satomi, S. Tanaka, S. Ryu, and S. Asano, "Field trial of GMPLS-based 40-Gbit/s wavelength path protection by photonic crossconnect with automatic chromatic dispersion compensation,"Proc. OFC2004, Paper WH3, March 2004.

[6] S. Matsumoto, T. Ohira, M. Takabayashi, K. Yoshiara, and T. Sugihara, "Tunable dispersion equalizer with a divided thin-film heater for $40 \mathrm{~Gb} / \mathrm{s}$ RZ transmissions," IEEE Photon. Technol. Lett., vol. 13, no. 8, pp. 827829, 2001.

[7] M. Yagi, H. Koyano, S. Ryu, and S. Satomi, "Application of automatic chromatic dispersion compensation for realization of all-optical network," Proc. OECC2003, Paper 15D4-4, Oct. 2003.

[8] M. D. Feuer, H. Sun-Yuan, S. L. Woodward, O. Coskun, and M. Boroditsky, "Electronic dispersion compensation for a $10-\mathrm{gb} / \mathrm{s}$ link using a directly modulated laser, "IEEE Photon. Technol. Lett., vol. 15, no. 12, pp. 1788-1790, 2003.

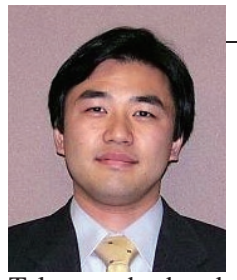

Mikio YAGI

Mikio Yagi received the M.E. degree in Electronics and Information Science Engineering from Chiba University, Japan in March 2000. He joined Japan Telecom Co., Ltd. in April 2000. In the Information and Communication Laboratories of Japan Telecom, he has been engaged in research on wavelength-division multiplexed (WDM) transmission systems at $40 \mathrm{Gbit} / \mathrm{s}$ and next-generation photonic networks.

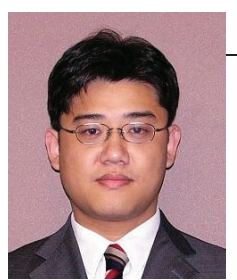

\section{Shinya TANAKA}

Shinya Tanaka received the M.E. degree in Information Engineering from University of Tokyo, Tokyo, Japan in March 2000. He joined Japan Telecom Co., Ltd. in April 2000. In the Information an Communication Laboratories of Japan Telecom, he has been engaged in research of control plane technologies of next generation photonic network systems.

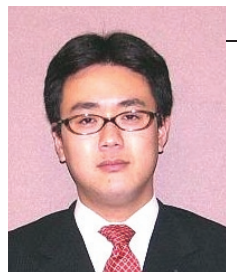

\section{Shuichi SATOMI}

Shuichi Satomi received the B.E. and M.E. degrees both in Information and Communication Engineering from Tokyo Denki University, Japan in 1999, 2001, respectively. He joined JapanTelecom Co., Ltd. in April 2001. In the Information and Communication Laboratories of JapanTelecom, he has been engaged in research on all-optical regeneration system and next generation photonic networks.

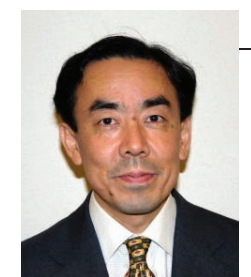

\section{Shiro RYU}

Shiro Ryu received the B.S., M.S., and $\mathrm{Ph} . \mathrm{D}$. degrees all in electronic engineering from the University of Tokyo, Tokyo, Japan in 1981,1983 , and 1993, respectively. He joined KDD in 1983. In KDD R\&D Labor atories, he has been engaged in the research activities on coherent optical fiber communication systems in conjunction with technologies including optical measurement, optical submarine cable systems, optical amplifiers, and wavelength-division multiplexing. He then moved to Japan Telecom in 2000. In Information and Communication La boratories of Japan Telecom, he is currently leading a research group regarding the next generation photonic network and systems. 


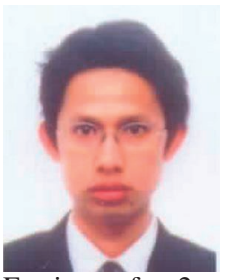

\section{Ahmad Suffian MOHAMAD}

Ahmad Suffian received his B.Eng. and M.Eng. degrees in Electronics Engineering from Shibaura Institute of Technology, Tokyo, Japan in 1999 and 2001, respectively. In 2001 he joined Malaysia premier ISP company and has served as ISP Technical Engineer for 2 years before decided to further his studies. Currently he is a PhD Candidate of Department of Information and Communication Engineering, Graduate School of Information Science and Technology, The University of Tokyo. His current researches are mainly focused on Optical Network Architecture. He is a student member of IEEE and IEICE of Japan.

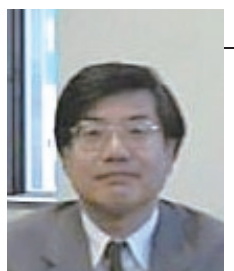

\section{Susumu YONEDA}

Susumu Yoneda received $\mathrm{Ph}$. D degree in system engineering from The Johns Hopkins University in 1984. He joined Bell Communications Research as a Member of Technical Staff in 1984, and worked on various data communication network including ATM. In 1993, he moved to the development department of Japan Telecom in Japan. He established the ATM network and services of Japan Telecom. He became the manager of Information and Communication Laboratory of Japan Tele com in 1997, and became the vice head of the laboratory in 2002. He also held the visiting professor position in National Institute of Informatics from 2002.

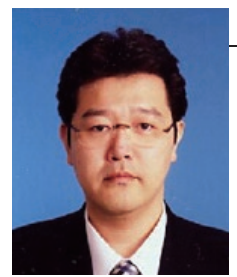

Takayuki FUJINO

received the Ph.D. degree in engineering from Meiji University in 1994. He joined the National Center for Science Information Systems (NACSIS) as an Assistant Professor. He has been an Assistant Professor at Infrastructure Systems Research Division, National Institute of Informatics (NII) since 2004. His research focuses on photonic network architecture.He is a member of IEICE, IPSJ, ISOC.

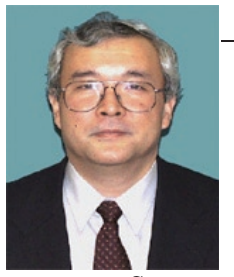

Shoichiro ASANO

Shoichiro Asano had graduated at Electronic Engineering, Faculty of Engineering, University of Tokyo, in 1970. He received ME and DE, both from University of Tokyo, in 1972 and 1975 respectively. Dr. Asano is a Director and Professor, Infrastructure Systems Research Division, National Institute of Informatics (NII) and a Professor, Graduate School of Information Science and Technology, University of Tokyo. His current researches are mainly focused on Optical Network Architecture and development of SuperSINET in Japan. 